The incidence of melanoma is increas ing steadily both in Poland and worldwide. Until 2010 three drugs were approved for the treatment of metastatic melanoma - dacarbazine (DTIC) in Europe and USA, fotemustine in Europe and interleukin-2 (IL-2) in USA. Approval of ipilimumab and vemurafenib in Europe and USA has changed the standard of care, while the next candidates such as dabrafenib and trametinib have improved survival in phase III studies in metastatic melanoma patients. An encouraging treatment strategy is the combination of dabrafenib and trametinib, evaluated in a phase I/II study with an ongoing phase III trial. Another promising new immune modulating monoclonal antibody (mAb) is anti-PD1 (BMS-936558), tested in an early phase trial in monotherapy or in combination with a multipeptide vaccine in metastatic melanoma patients. Ipilimumab or BRAF inhibitors (vemurafenib, dabrafenib) seem to be active in patients with brain metastases. Intensive research of melanoma vaccines is currently being carried out in a number of countries worldwide. However, no vaccine in the treatment of melanoma has been approved by regulatory authorities so far. Lack of effective therapy in patients with high-risk resected melanoma led to a number of clinical studies of adjuvant treatment. Interferon- $\alpha$ (INF- $\alpha$ ) therapy in this setting is still controversial. A dendritic cell-based vaccine in a randomized phase II trial showed a survival benefit over the control group in patients with high-risk resected melanoma. Promising results of longterm survival of advanced resected melanoma patients in a phase II study evaluating the genetically modified tumour vaccine (GMTV) AGI-101 were reported.

This review provides an update on clinical strategies used or tested in patients with metastatic melanoma.

Key words: melanoma, BRAF inhibitor, immunotherapy, anti-CTLA4, cancer vaccines.

\section{What is new in the treatment of advanced melanoma? State of the art}

\author{
Jacek Mackiewicz
}

Department of Cancer Immunology, Chair of Medical Biotechnology, Poznan University of Medical Sciences, Greater Poland Cancer Centre, Poznan, Poland

\section{Introduction}

The incidence of melanoma is increasing steadily both in Poland and worldwide. Melanoma presents the highest death rate among young people between 20 and 29 years of age. The mortality to incidence ratio in Poland is much higher than in Western Europe [1]. More than 2500 skin melanomas were diagnosed in Poland in 2009. Over 1000 patients will die each year due to metastatic disease [2]. Thus, there is a critical need to improve the understanding, prevention, and treatment of this malignancy.

Until 2010 three drugs were approved for the treatment of metastatic melanoma - dacarbazine (DTIC) in Europe and USA, fotemustine in Europe and interleukin-2 (IL-2) in USA. However, none of these drugs showed beneficial effects on survival of patients in phase III trials. Although objective responses after standard treatment are being observed, as well as a few long-term remissions after IL-2 (less frequently after chemotherapy), no predictive factors for these treatment strategies are known. Multidrug chemotherapy consisting of DTIC (BOLD, CVD, Dartmouth) results in a higher response rate, although is not beneficial in terms of survival over DTIC alone. Also commonly used temozolomide or paclitaxel with or without carboplatin did not result in overall survival (OS) prolongation [1]. In addition, various strategies of combining chemotherapy with biotherapy did not bring significant benefits to patients [3].

Recent approval of ipilimumab and vemurafenib in Europe and USA changed the standard of care of metastatic melanoma patients. Moreover, positive results of phase III trials evaluating dabrafenib and trametinib may lead to approval of these drugs in the near future. A number of new small molecules or immunotherapy strategies are currently in various stages of clinical development in metastatic melanoma.

Lack of effective treatment in patients with high-risk resected melanoma led to a number of clinical trials. Several randomised phase III studies evaluating interferon (IFN)- $\alpha-2 a$ and IFN- $\alpha-2 b$ in low, medium and high doses have been carried out. Only in two of them was a statistically significant improvement of OS observed. High-dose IFN- $\alpha-2 b$ (Intron ${ }^{\circledR}$ ) has been approved by the U.S. FDA (Food and Drug Administration) based on the results of the ECOG 1684 trial. At a median follow-up of 6.9 years the study demonstrated a statistically significant improvement in survival for patients treated with IFN- $\alpha-2 b$ compared to the control group. However, at 12.6 years of follow-up, OS was not significantly different between the two study groups. Intron is indicated in patients after resection of high-risk melanoma (stage IIB and stage III). Recently (March 2011) pegylated-IFN- $\alpha-2 b$ (Sylatron ${ }^{\circledR}$ ) has been approved for the treatment of patients with melanoma with microscopic or gross nodal involvement after definitive surgical resection including complete lymphadenectomy. The approval was based on the results of the EORTC 18991 trial. The study demonstrated a lack of survival benefit with the improvement in recurrence-free survival (RFS) in unselected patients treated with Sylatron compared to the placebo control [4]. 
Despite approval of IFN in the adjuvant treatment of melanoma its use in the clinic is limited due to the high toxicity and questionable effectiveness. Currently we are awaiting the results of two melanoma adjuvant phase III studies evaluating ipilimumab and MAGE-A3 ASCI vaccine $[5,6]$.

\section{Kinase inhibitors}

In recent years genetic and molecular studies and a number of somatic mutations playing a key role in melanoma pathogenesis have been identified. Moreover, understanding the underlying mechanisms leading to melanoma cell progression resulted in the development of targeted therapies in the treatment of melanoma patients. The best defined mutations are in oncogenes, NRAS, BRAF, c-KIT, GNAQ, GNA11 and suppressor genes such as PTEN or P53. Very recently driver mutations in PPP6C, RAC1, SNX31, TACC1, STK19, and ARID genes were described in UV (sun) dependent melanoma [7].

\section{BRAF inhibitors}

BRAF is a member of the Raf family of serine threonine kinases (ARAF, BRAF, CRAF) which are part of the Ras/ Raf/MEK/ERK mitogen-activated protein kinase (MAPK) signalling pathway. Activation of the MAPK pathway results in increased transcription of genes required for cell cycle entry. $B R A F$ mutations are identified in $40-60 \%$ of melanomas. The most common is V600E, which occurs in $80 \%$ of BRAF mutant melanoma cells. Less frequent are V600K and V600 D/R, identified in $16 \%$ and $3 \%$, respectively [9]. BRAF mutation usually occurs in younger patients ( $<55$ years) with the localization of primary melanoma on the trunk. BRAF mutation is not associated with constant sun exposure but is more often related to frequent solar burns during childhood [10-12]. BRAF mutation is also a prognostic factor linked with a poorer survival (8.5 vs. 5.7 months in BRAF wild-type and BRAF mutant melanoma, respectively) [9].

Sorafenib, a multikinase inhibitor, was the first RAF inhibitor tested in clinical trials. Sorafenib inhibits not only mutated BRAF but also BRAF wild type and C-KIT mutated melanoma as well as PDGFR (platelet-derived growth factor

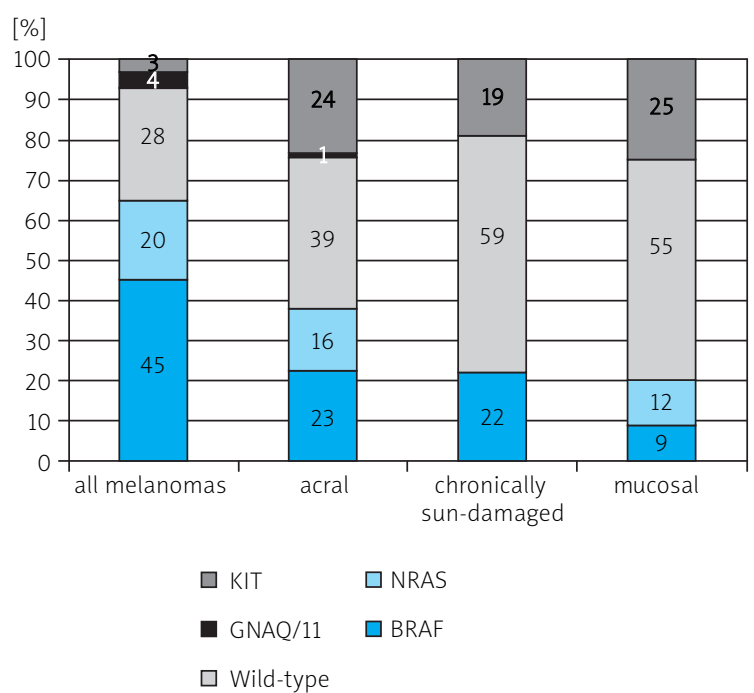

Fig. 1. Graph demonstrating various genetic subgroups of melanoma [8] receptor) and VEGFR (vascular endothelial growth factor receptor) -2 and -3 [13]. However, sorafenib in combination with carboplatin and paclitaxel did not show an advantage over chemotherapy in a phase III study.

The only BRAF inhibitor approved so far by the U.S. FDA and EMA (European Medicine Agency) in the treatment of metastatic melanoma is vemurafenib. This selective BRAF inhibitor has been tested in a phase 2 trial (BRIM2) in patients with metastatic melanoma with confirmed BRAF V600E mutation after progression of earlier systemic treatment. The observed overall response rate was 53\% [6\% with a complete response $(C R)$ and $47 \%$ with a partial response $(P R)]$. The median duration of response was 6.7 months. Primary progression was observed only in $14 \%$ of patients. Some patients responded after receiving vemurafenib for more than 6 months. The median OS was 15.9 months [14]. Vemurafenib was also evaluated in a phase 3 trial (BRIM3) which led to its approval by the FDA in August 2011 and by the EMA in February 2012. In the BRIM3 trial vemurafenib was studied as the first line treatment in metastatic melanoma patients with BRAF V600E mutation. 675 patients were randomly assigned to the vemurafenib treatment arm (960 mg twice daily) or DTIC control arm (1000 mg/m² every 3 weeks). At the time of study analysis the objective response rate was higher in patients receiving vemurafenib (48\% vs. $5.5 \%$ ). The median progression-free survival (PFS) was longer in patients treated with the study drug [5.3 vs. 1.6 months; hazard ratio (HR) 0.26; 95\% confidence interval (CI) 0.20-0.38; $p<0.001]$. HR for death in the vemurafenib group was 0.37 (95\% Cl: $0.26-0.55 ; p<0.001)$. At 6 months, OS was $84 \%$ for patients receiving vemurafenib and $64 \%$ for those treated with DTIC. Clinical benefit in patients receiving vemurafenib was independent of age, gender, ECOG (Eastern Cooperative Oncology Group) performance status, stage or level of LDH (lactate dehydrogenase). Generally treatment with vemurafenib was well tolerated. The most common adverse events (AEs) were grade 1 or 2 and included arthralgia, rash, photosensitivity, nausea, fatigue and alopecia. Cutaneous squamous-cell carcinoma or keratoacanthoma was diagnosed in $26 \%$ of patients participating in BRIM2 and $18 \%$ in the BRIM3 study $[14,15]$. Updated OS results of the BRIM3 study have been presented at the 2012 ASCO (American Society of Clinical Oncology) annual meeting. The overall response rate in patients treated with vemurafenib was 57\% (5.6\% - CR, 51.3\% - PR) compared with 8.6\% (1.2\% - CR, 7.4\% - PR) observed in patients receiving DTIC. Median PFS at this time point of the study was 6.9 months in patients treated with the study drug and 1.6 months in patients receiving chemotherapy (HR 0.38; 95\% Cl: 0.32-0.46; $p<0.001)$. The median OS was also statistically longer in patients treated with vemurafenib (13.6 vs. 9.7 months; HR 0.70; 95\% Cl: 0.57$0.87 ; p<0.001)$. The earlier analysis of the BRIM3 study demonstrated $63 \%$ reduction of risk of death, while the updated results showed a 30\% risk reduction. The study also demonstrated lower OS benefit in stage IIIc and IV-M1a/M1b than M1c in patients treated with vemurafenib compared to DTIC. These differences might be due to the higher number of patients treated with ipilimumab in the DTIC group after the study completion ( $26 \%$ vs. $18 \%$ ). In patients treated with 
vemurafenib, adverse cutaneous skin carcinoma, keratoacanthoma and skin papilloma were noted respectively in 19\%, $11 \%$ and $28 \%$ of patients [16].

Recently, results of an open-label, multicenter safety study of vemurafenib in patients with metastatic melanoma were presented. Of 1964 screened patients, 914 were enrolled in the study and 834 were evaluable for toxicity analysis. $30 \%$ of patients did not receive any prior systemic treatment due to the metastatic melanoma. AEs were observed in $66 \%$ of patients and were mainly related to vemurafenib treatment. The most frequently observed AEs of any grade were arthralgia (31\%), rash (29\%), fatigue (22\%), photosensitivity (21\%) and nausea (15\%). 33\% and $1.9 \%$ of patients developed grade 3 and 4 AEs, respectively. The most frequently observed were rash (3.6\%), arthralgia (3.1\%) and cutaneous cell carcinoma/keratoacanthoma (4.3\%). In 6\% of patients treatment was discontinued due to AEs (mainly arthritis and abdominal pain). At the time of study analysis 302 patients were evaluable for tumour assessment at week 8 of treatment; $61 \%$ developed objective responses, and $29 \%$ stable disease (SD) [17].

Another active BRAF kinase inhibitor is dabrafenib (GSK2118436), evaluated in a randomized, open-label, multicenter phase 3 study (BREAK-3) in patients with BRAF V600E mutated metastatic melanoma. In the study arm previously untreated patients received oral dabrafenib at a dose of $150 \mathrm{mg}$ twice a day, while in the control arm they received DTIC at a dose of $1000 \mathrm{mg} / \mathrm{m}^{2}$ every three weeks. ECOG performance status greater than 1 was noted in $31 \%$ of patients and $66 \%$ had stage M1c melanoma. The objective response rate was higher in patients treated with dabrafenib - 53\% vs. $19 \%$. Patients in the study arm had longer median PFS -5.1 vs. 2.7 months (HR 0.30; $95 \% \mathrm{Cl}: 0.18-0.53 ; p<0.0001$ ). The OS data were immature for analysis at this point of the study. The most common AEs noted in patients treated with dabrafenib were hyperkeratosis (37\%), headache (32\%), pyrexia (28\%), arthralgia (27\%) and skin papillomas (24\%). Grade 3 and 4 toxicity included pyrexia (6\%), squamous cell carcinoma (6\%) and new primary melanoma (2\%) [18].

Vemurafenib compared to dabrafenib more frequently caused photosensitivity, while dabrafenib caused pyrexia refractory to antipyretics.

A number of clinical trials have shown low efficacy of anticancer agents in melanoma patients with brain metastases. However, small molecules have demonstrated some efficacy in patients with solid tumours with concomitant brain metastases.

Dabrafenib demonstrated high clinical efficacy in patients with BRAF V600E/K mutation with intracranial lesions. In a phase 2 study (BREAK-MB) stage IV melanoma patients with $\geq 1$ intracranial metastases were enrolled. 127 patients were recruited to one of the two study arms, but only 41 patients reached 8-week disease assessment at the time of interim analysis. Patients in cohort A did not receive any prior brain metastasis treatment before entering the trial. Patients in group $\mathrm{B}$ before enrolment developed intracranial progression following prior brain therapy. A 53\% unconfirmed overall intracranial response rate (OIRR) was reached in patients with the BRAF V600E mutant in both study cohorts. The uncon- firmed OIRR was $20 \%$ and $50 \%$, respectively, in arms $A$ and $B$. These preliminary results confirm efficacy of dabrafenib in melanoma patients with intra- and extracranial metastases with acceptable toxicity [19].

\section{MEK inhibitors}

The observation that nearly all melanomas demonstrate constitutive MAPK activity led to the development of smallmolecule MEK inhibitors, such as PD0325901, selumetinib (AZD6244) and Cl-1040, tested in an unselected group of melanoma patients. Initial studies evaluating these MEK inhibitors were disappointing, limiting their further evaluation mainly due to high toxicity [20]. Interest in the clinical development of MEK inhibitors was renewed by the development of a reversible, highly selective allosteric inhibitor of MEK 1/2 - GSK1120212 (trametinib), tested in a phase 3 (METRIC) study. In the study arm BRAF V600/K mutant advanced or metastatic melanoma patients were treated with trametinib, while in the control arm patients were treated with chemotherapy (paclitaxel/DTIC). Patients on chemotherapy were allowed to cross over to the trametinib arm after disease progression. The overall response rate was greater in patients treated with trametinib $-24 \%$ vs. $7 \%$. In the group receiving trametinib the median PFS was longer than in the control arm - 4.8 vs. 1.4 months (HR 0.44; 95\% Cl: 0.31-0.64; $p<0.0001)$. The 6 -month OS in the trametinib group was $81 \%$ compared with $67 \%$ in the chemotherapy group (HR 0.53; $95 \% \mathrm{Cl}: 0.30-0.94 ; p<0.01)$. The most frequently observed adverse events in patients treated with trametinib included skin rash, diarrhoea, oedema, hypertension and fatigue. Characteristic AEs associated with MEK inhibitor treatment included chorioretinopathy $(<1 \%)$ and decreased ejection fraction (7\%) [21].

\section{Overcoming resistance to BRAF inhibitors}

BRAF inhibitors induce spectacular tumour shrinkage in patients with BRAF mutant melanoma, although these responses are short-lived due to the secondary resistance to the drug observed in nearly all treated patients (median PFS around 7 months) [20]. A number of potential BRAF inhibitor resistance mechanisms have been reported, which mostly depend on a common set of signalling pathways. Basic studies have already demonstrated that reactivation of MAPK signalling is usually related to vemurafenib resistance. Combination of MEK and BRAF inhibitors was effective at overcoming the resistance mediated by MEK1 mutations, COT overexpression, BRAF truncation and acquired Ras mutations [22-25].

A combination of dabrafenib (BRAF inhibitor) and trametinib (MEK inhibitor) was tested in a phase I/II study in 77 BRAF V600 mutant metastatic melanoma patients. The observed overall response rate was $56 \%$ with 4 CR, 39 PR, 29 SD and 3 PD. Overall PFS was 7.4 months. Pyrexia (6.5\%), fatigue (6.5\%) and dehydration (6.5\%) were the most commonly noted serious AEs. Dabrafenib combined with trametinib was associated with a lower incidence of MEK inhibitor related rash and BRAF inhibitor induced hyperproliferative skin lesions when compared to the single agent treatment. Skin toxicity over grade 2 occurred in near- 
ly $2 \%$ of patients. Cutaneous squamous cell carcinoma and keratoacanthoma were observed in $2 \%$ of patients [26].

\section{C-KIT inhibitors}

Activating mutations in KIT have been discovered in acral melanomas and melanomas arising from mucosal or chronically sun-damaged sites. Previously KIT was believed to function as a tumour suppressor, but further research suggested that in certain contexts, KIT functions as an oncogene. KIT encodes type III transmembrane receptor tyrosine kinases. Three subsequent phase II studies evaluating imatinib in metastatic melanoma patients with KIT mutation or amplification have been conducted. Imatinib is a tyrosine kinase receptor inhibitor, which selectively inhibits the tyrosine kinases of the bcr-abl, c-KIT, and PDGFR (platelet-derived growth factor receptors). Findings in these studies are consistent, with the observed objective response rate around 25\%. Interestingly, responses to imatinib treatment were observed only in patients with the KIT mutation located in exons 11 and 13 [8].

Recently results of a phase II study testing dasatinib in patients with advanced or metastatic mucosal, acral and solar melanomas have been presented. Dasatinib's mechanism of action is similar to imatinib. Out of 57 enrolled patients, KIT status assessment was performed in 42 cases with only 3 presenting KIT mutation. An objective response was observed in $7 \%$ of patients and SD was noted in $25 \%$. The second stage of this trial will enrol only patients with KIT mutation [27].

The only randomized phase III trial conducted in KIT mutant melanoma was initiated in 2010. The study evaluated efficacy of nilotinib compared with DTIC [28]. However, challenging accrual due to uncommon KIT mutation in melanoma forced the sponsor to modify this study to a single arm phase II trial assessing nilotinib alone. These results of drugs for melanomas harbouring the KIT mutation need further evaluation. Currently additional agents targeting KIT and evaluating KIT inhibitors in combinations with other drugs are ongoing [8].

\section{Immunotherapy}

\section{Anti-CTLA-4}

Another new drug approved for the treatment of metastatic melanoma is ipilimumab (Yervoy $\left.{ }^{\circledR}\right)$. I pilimumab is a fully human monoclonal antibody (mAb) targeting cytotoxic T-lymphocyte antigen-4 (CTLA-4). CTLA-4 is an immune checkpoint molecule that is up-regulated on activated T-cells. It suppresses further activation of specific CD4+ and CD8+ Tcells by interaction with dendritic cells (DCs) or directly as a result of a contact between suppressor and effector T lymphocytes. The anti-CTLA4 mAb by blocking the interaction of CTLA-4 with CD80/86 switches off the mechanism of immune suppression and enables continuous, unrestrained stimulation of T-cells by DCs [1]. Two IgG mAb directed against CTLA-4 - ipilimumab and tremelimumab - have been tested in number of clinical trials in patients with melanoma. Ipilimumab was first approved in the U.S. (2010) and subsequently in Europe (2011) for the second line treatment after failure of chemotherapy. Ipilimumab is also accepted for treatment of previously untreated patients with metastatic melanoma (only in the U.S.) The approval was based on the results of a randomized phase III trial, which included 676 HLA-A*0201-positive patients with unresectable stage III or IV melanoma. Patients enrolled were previously treated with IL-2 or chemotherapy and were then randomly assigned to ipilimumab plus peptide (gp100) vaccine (403 patients), ipilimumab alone (137), or gp100 alone (136) study groups. Ipilimumab, at a dose of $3 \mathrm{mg} / \mathrm{kg}$, was administered with or without gp100 every 3 weeks for up to four treatments. Treatment with ipilimumab was associated with a 32\% and 34\% reduction of death-related risk when administered with gp100 or in monotherapy. Patients receiving ipilimumab alone or in combination with the peptide vaccine had a nearly identical median OS of 10 and 10.1 months, compared with 6.4 months in patients receiving gp100 alone $(p<0.001)$. Twoyear OS was observed in $23 \%$ of patients treated with ipilimumab, and in 14\% in the control arm [29]. Ipilimumab was also tested in a phase III trial in previously untreated metastatic melanoma patients. In the study arm patients received $10 \mathrm{mg} / \mathrm{kg}$ ipilimumab with DTIC while in the control arm they received DTIC alone. OS was longer in patients treated with the study drug (11.2 vs. 9.1 months; $\mathrm{HR}=0.72$; $p=0.0009$ ). Two-year OS was observed in $28.5 \%$ of patients receiving ipilimumab and $17.9 \%$ in the control arm. The threeyear OS rate was $20.8 \%$ in patients of the study arm and $12.2 \%$ in the DTIC group [30]. New OS data from two phase II studies, CA184-008 and CA184-22, in patients treated with $10 \mathrm{mg} / \mathrm{kg}$ ipilimumab demonstrated a 2-year OS rate of 30\% and $24 \%$ and a 3 -year OS rate of $25 \%$ and $24 \%$ respectively [31, 32].

Treatment with ipilimumab causes so-called immunerelated adverse events (irAE), which occur in $80 \%$ of patients. Grade 3-5 irAE were observed in 7-13\% of patients treated with $3 \mathrm{mg} / \mathrm{kg}$ ipilimumab, while a higher $10 \mathrm{mg} / \mathrm{kg}$ dose caused grade 3-4 toxicity in 22-39\% of patients [33-36]. The most frequently observed irAE were diarrhoea, colitis, endocrinopathies, dermatitis and hepatitis. These irAE specific for anti-CTLA-4 are quite easy to manage using glucocorticosteroids [37]. At the 7th International Melanoma Congress in 2010 it was reported that when the ipilimumab treatment was preceded by administration of GM-CSF-gene modified autologous cell melanoma vaccine, grade 3 and 4 irAE were not observed. These results indicate that future effective ipilimumab therapy may require concomitant induction of specific anti-melanoma $T$ cell clones [38].

The objective response rate observed in phase II and III studies in patients treated with ipilimumab was 7-15\% [39]. Responses were correlated with irAE [40]. 36\% of patients with grade 3 and 4 toxicity developed objective responses, while in patients without irAE responses occurred in 5-11\% [41]. Median time to response was 12 weeks with a median duration of 11.5 months [42]. $68 \%$ of patients responded after 12 weeks from the beginning of the treatment with ipilimumab. Retrospective analysis of two phase II studies (CA 184-022 and CA 184-008) demonstrated that 39\% of patients with disease progression according to modified WHO criteria benefited from the ipilimumab treatment and their 
tumour assessment might be evaluated as PR or SD using immune-related response criteria (irRC) [43].

During the 2012 ASCO meeting results of the ipilimumab U.S. expanded access program (EAP) in patients with unresectable stage III or IV melanoma were presented. The study also enrolled patients with brain metastases (27\%), ocular melanoma (5\%) and mucosal melanoma (4\%). 906 patients were treated with ipilimumab at a dose of $10 \mathrm{mg} / \mathrm{kg}$ every 3 weeks (4 doses - induction phase) followed by $10 \mathrm{mg} / \mathrm{kg}$ every 12 weeks (maintenance phase) until progression or unacceptable/unmanageable toxicity of the treatment. Durable OS over 3 years was observed in $17 \%$ of patients. IrAE were noted in $27 \%$ of patients, while $11 \%$ of patients discontinued treatment due to drug toxicity [44]. Currently a randomized, double-blind, phase III trial comparing two doses of ipilimumab, $3 \mathrm{mg} / \mathrm{kg}$ vs. $10 \mathrm{mg} / \mathrm{kg}$, in metastatic melanoma patients has finished enrolment [45].

Treatment with vemurafenib following ipilimumab therapy seems to be associated with hypersensitivity skin reactions in patients with metastatic melanoma. Rash associated with vemurafenib was observed in 13 out of 16 treated patients (81\%). Four patients developed grade 3 maculopapular rash which occurred 8 days following initiation of the vemurafenib treatment. Biopsies demonstrated spongiotic and perivascular dermatitis with eosinophils consistent with a drug hypersensitivity reaction. Progression towards life-threatening reactions such as anaphylaxis or Stevens-Johnson syndrome which would require discontinuation of vemurafenib treatment was not observed. The incidence of grade 3 rash was higher than observed in patients treated with vemurafenib in the phase III BRIM3 trial (25\% vs. $8 \%$; $p=0.02)$ [46]. Currently a phase I/II trial evaluating ipilimumab in combination with vemurafenib is active with the first enrolled metastatic melanoma (BRAF V600 mutant) patients in the fourth quarter of 2011 [47].

Ipilimumab has also shown some activity in patients with metastatic melanoma and brain metastases, particularly when metastases were small and asymptomatic. In a recently published phase II study a $24 \%$ and $10 \%$ intracranial response rate was observed, respectively in patients neurologically asymptomatic without concomitant corticosteroid treatment and with neurological symptoms on a stable dose of corticosteroids [48].

Ipilimumab was also evaluated in combination with fotemustine (NIBIT-M1 trial) in 86 patients with asymptomatic brain metastases (7 patients had undergone earlier whole brain radiotherapy or radiosurgery). Four doses of $10 \mathrm{mg} / \mathrm{kg}$ ipilimumab were administered every 3 weeks with a weekly $100 \mathrm{mg} / \mathrm{m}^{2}$ dose of fotemustine for 3 weeks, followed by ipilimumab every 12 weeks (from week 24) combined with fotemustine every 3 weeks (from week 9). The immune-related (ir) disease control rate (irDCR $=C R+P R+S D$ using the ir response criteria) was $50 \%$ and the immune-related overall response rate (irORR $=\mathrm{CR}+\mathrm{PR}$ ) was 40\%. Median irPFS was 4.6 months and 1 -year OS was $52 \%$. Median OS was not reached at the time of study analysis. $60 \%$ of patients developed grade 3 or 4 toxicity (haematological toxicity - 50\%, elevated ALT/AST - 5\%, gastrointestinal adverse events - 5\%) [49].

Tremelimumab is another mAb targeting CTLA-4. It was administered in phase $\mathrm{II}$ at the dose of $15 \mathrm{mg} / \mathrm{kg}$ every
3 months to previously treated patients. In $8.3 \%$ of 256 metastatic melanoma patients enrolled, objective clinical responses were observed, while the median OS was 10.2 months [50]. In the phase III trial 643 patients were treated with tremelimumab in monotherapy or with DTIC/temozolomide (TMZ) in the first line setting. Analysis of preliminary results failed to show the advantage of tremelimumab over the standard therapy (OS 11.8 vs. 10.7) and the trial was terminated [51]. Treatment effectiveness of tremelimumab in combination with high doses of interferon- $\alpha-2 b$ was evaluated in a small phase II trial which enrolled only 16 patients with inoperable stage III and IV melanoma. A clinical response was observed in 19\% of patients. The most frequent grade 3 and 4 adverse events included neutropenia in 3 patients (19\%), elevated liver enzymes in 2 (13\%), fatigue in $6(38 \%)$ and anxiety in 2 (13\%) [52].

\section{Anti-PD-1}

Another human mAb modulating the immune system is BMS-936558 (MDX-1106) directed against the programmed death-1 receptor (PD-1R), the ligand of which (PD-1L) can be directly expressed on melanoma cells. PD-1R is a part of the B7:CD28 family of co-stimulatory molecules that regulate T-cell activation and tolerance, and thus anti-PD-1R can play a role in breaking tolerance [53]. BMS-936558 was tested in 95 metastatic melanoma patients undergoing earlier systemic therapy. The study drug was administered intravenously every 2 weeks until PD or CR, for a maximum of 12 cycles. The doses varied depending on the study cohort $(0.1-10 \mathrm{mg} / \mathrm{kg})$. Grade 3 and 4 toxicity was observed in 19\% of patients, mainly including gastrointestinal (4\%), endocrine (2\%) and hepatobiliary disorders (1\%). The objective response rate was 20$41 \%$ depending on the study cohort. Of the 20 patients who responded to the treatment, 12 developed a response lasting over 1 year. BMS-936558 is currently being evaluated in further clinical trials [54]. BMS-936558 was also tested with the combination of a multipeptide vaccine in a phase I study in 30 previously treated metastatic melanoma patients. The vaccine consisted of MART-1/gp100/NY-ESO1 peptides with adjuvant Montanide ISA 51. In all study cohorts patients responded to the treatment $(1 / 3 / 10 \mathrm{mg} / \mathrm{kg}-$ 2PR/5PR/2PR and 1 SD). Immunological tests demonstrated decreased PD-1 receptors on CD4+ and CD8+ lymphocytes, decreased CTLA4 receptors on CD8+ and increased CTLA4 receptors on CD4+ cells [55].

\section{Other agents modulating the immune system}

The mAb BMS-663513 targeting co-stimulating molecule CD137 (4-1BB) acts according to a different mechanism. Binding of the ligand or anti-CD137 antibody with 4-1BB receptor on the surface of $\mathrm{T}$ lymphocytes provides a co-stimulating signal enhancing the cell's activation and triggering its proliferation. The phase I trial enrolling 54 patients with solid tumours has shown an acceptable toxicity level and a certain clinical activity of BMS-663513 [56]. We look forward to the results of a large randomized phase II study which has just been completed [57]. CP-870.893 is a human agonistic $\mathrm{mAb}$ to co-stimulating molecule CD40 that is up-regulated on the surface of the antigen-presenting cells (APCs). 
A phase I trial has shown PR in $4(27 \%)$ out of 15 patients with advanced melanoma and 1 CR lasting 18 months after single administration of the drug [58]. Currently, the trial evaluating the efficacy of CP-870,893 in combination with carboplatin and paclitaxel has been completed and the results probably will be disclosed soon [59].

\section{Cancer vaccines}

Whole tumour cell vaccines and stimulating adjuvants were among the first and fundamental specific tumour immunotherapy strategies. In 1990 Berd and colleagues tested autologous melanoma vaccine with BCG (Bacillus Calmette-Guerin) in patients with metastatic melanoma. The median OS observed in these patients was 10 months. The next generation of cancer vaccines consisted of established cell lines (allogeneic vaccines) which present antigens specific for a given tumour type. The immunogenicity of allogeneic vaccines is improved by the response to alloantigens expressed on the vaccine cells. Allogeneic vaccines have superseded autologous vaccines due to the difficulties in obtaining a sufficient number of cells for repeated vaccinations [3]. An example of allogeneic polyvalent cancer vaccine is Cancervax, consisting of three established melanoma cell lines and BCG as an adjuvant. After encouraging results of a phase II study, Cancervax did not improve survival in a phase III trial in metastatic melanoma patients [60]. Encouraging results of a phase $\|$ study evaluating Melacine led to a phase III study in patients with metastatic melanoma. Melacine is a melanoma tumour cell lysate vaccine composed of two allogeneic melanoma cell lines (MSM-M-1 and MSMM-2) combined with Detox ${ }^{\circledR}$ adjuvant [61]. Melacine did not improve survival in vaccinated patients compared to the control group. However, retrospective analysis showed that patients receiving Melacine and expressing at least two of five human leukocyte antigens (HLA) present on the vaccine cells developed longer RFS and OS ( $p=0.0002$ and $p=0.0001$, respectively). For that reason, the HLA pattern of the patient served here as a biomarker and allowed stratification of patients who would respond to the treatment [62].

Mackiewicz et al. presented the results of two phase II clinical studies (trials 3 and 5) conducted in almost 200 patients after resection of stage IIIB, C and IV melanoma [63]. In both studies patients were vaccinated with only one in the class of allogeneic genetic vaccines AGI-101 composed of two irradiated melanoma cell lines modified to express Hyper-IL-6 - a fusion protein composed of interleukin 6 (IL-6) and soluble IL-6 receptor. AGI-101 ( $5 \times 10^{7}$ cells per dose) was administered 8 times at 2-week intervals (induction phase) and then monthly (maintenance phase). At disease progression the induction phase (+/- surgery) was restarted, followed by a second maintenance phase. At progression 43 (trial 3) and 39 (trial 5) patients were re-induced $+/$ - surgery followed by a second maintenance phase; of those 11 and 16 patients respectively are alive following re-induction. Disease-free survival (DFS) probability at 5 years for trials 3 and 5 was $54.8 \%$ and $40.6 \%$ for stage IIIB, $25.0 \%$ and $24.0 \%$ for IIIC, and $8.5 \%$ and $17.7 \%$ for IV. The 5 -year survival in trial 3 was $66.7 \%, 43.8 \%$ and $26.1 \%$ respectively in stage IIIB, IIIC and IV. In trial 5 the 5 -year survival was as follows: $56.3 \%, 39.8 \%$ and $41.2 \%$ cor- respondingly in stage IIIB, IIIC and IV. The OS observed in trial 3 was 4.4 years and 3.1 years in trial 5. The vaccine was well tolerated as no vaccine-related toxicity of CTC $>2$ was detected [63]. In our studies the median DFS of treated patients was at least 3 times longer than control patients in three large randomized trials [64-66]. In the EORTC 18891 study, the median DFS of patients in the control arm was 1.6 and 0.64 years for stage IIIB and IIIC, respectively, with 33\% and $15.7 \%$ of patients surviving 4 years [64]. In patients with stage IIIB, IIIC and IV, Eigentler et al. reported median DFS of 0.63 years and $15 \%$ of patients disease free at 5 years [65]. Bystryn et al. reported for placebo patients the median DFS of 0.64 years with $23 \%$ of patients disease free at 2 years [66]. Furthermore, in a recently presented meta-analysis including 33 trials evaluating survival in patients with resected and unresected stage IV melanoma, the 2-year OS rate observed in patients after surgical resection of metastases was only $27 \%$ [67].

Broad research on DCs demonstrated that they are the most efficient APCs $[68,69]$. DCs play a crucial role in inducing the immune response. They are the only representatives of APCs that are capable of inducing a primary response of virgin T lymphocytes. The use of DCs for antigen presentation offers an opportunity to trigger an immune response even to weakly immunogenic tumour antigens and break immune tolerance. A phase III study conducted in metastatic melanoma patients evaluated the efficacy of autologous DCs pulsed with peptides presented in the context of HLA class I and II. In the control arm patients were treated with DTIC. However, the study was terminated after preliminary analysis due to the lack of superiority of the vaccine over DTIC [70]. Nevertheless, only 53 patients in the vaccine group and 55 in the control arm were participating in the trial and the vaccine was administered depending on the amount of DC (two up to five times; only 14 patients received more than 6 doses). However, subsequent analysis demonstrated that vaccinated patients with HLA-A2 +/HLA-B44 haplotype showed longer survival than those treated with DTIC [71]. DC vaccine was also tested in patients with high-risk resected melanoma (stage III and IV). In one study arm 56 patients (stage III 46, IV - 10) were treated with autologous monocyte-derived DC vaccine primed with autologous tumour lysate. In the control group 53 patients (stage III - 47, IV - 5) underwent observation. At a median follow-up of 22 months DFS was significantly longer in vaccinated patients (HR 0.45; $95 \% \mathrm{Cl}$ : $0.29-0.69 ; p<0.05)$, but there was no difference in OS between the study arms (HR 0.71; 95\% Cl: 0.40-1.25; $p=0.23$ ). $60 \%$ of patients treated with the DC vaccine remained alive at the time of study analysis. The investigators observed a significant correlation between reduction of risk and vaccineinduced strong delayed type hypersensitivity reaction [72].

Intensive research on melanoma vaccines is currently being carried out worldwide. However, no vaccine in the treatment of melanoma has been approved by regulatory authorities so far.

\section{Future perspectives}

In recent years significant progress in the treatment of advanced melanoma has been seen. However, for further 
improvement identification of good response biomarkers is needed. Also we need to learn how to evaluate and identify responses that would eventually mean survival advantages. Likewise, management of drug resistance is a big challenge in melanoma treatment. Combination therapy including MEK and BRAF inhibitors in overcoming resistance to BRAF inhibitors in patients with BRAF mutated metastatic melanoma needs confirmation in upcoming phase 3 studies. Also development of successful BRAF inhibitor/immune therapy-based (anti-CTLA4 or anti-PD1 therapy) combinations offers the real possibility that very durable responses could be achieved. Likewise, strategies composed of immunomodulating agents and cancer vaccines may result in higher efficacy of the treatment with fewer adverse events related to drugs modulating the immune system.

\section{The author declares no conflict of interest.}

\section{References}

1. Mackiewicz J, Kwinta L. New targeted therapies in the treatment of patients with metastatic melanoma. Contemp Oncol 2010; 14: 15-22.

2. National Cancer Registry http://www.onkologia.org.pl/pl/p/11.

3. Maio M, Mackiewicz A, Testori A, et al.; Thymosin Melanoma Investigation Group. Large randomized study of thymosin alpha 1, interferon alfa, or both in combination with dacarbazine in patients with metastatic melanoma. J Clin Oncol 2010; 28: 1780-7.

4. Mackiewicz J, Mackiewicz A. Immunotargeting of melanoma, current management of malignant melanoma. Ming Y Cao (ed.). InTech 2011. Available from: http://www.intechopen.com/articles/show/ title/immunotargeting-of-melanoma.

5. http://www.clinicaltrials.gov/ct2/show/NCT00636168?term=ipilimumab+adjuvant+melanoma\&rank $=1 \mathrm{~A}$.

6. http://www.clinicaltrials.gov/ct2/show/NCT00796445?term= MAGE+DERMA+melanoma\&rank=2.

7. Hodis E, Watson IR, Kryukov GV, et al. A landscape of driver muta tions in melanoma. Cell 2012; 150: 251-63.

8. Postow MA, Carvajal RD. Therapeutic implications of KIT in melanoma. Cancer J 2012; 18: 137-41.

9. Davies H, Bignelli GR, Cox C et al. Mutations of the BRAF gene in human cancer. Nature 2002; 417: 949-54

10. Curtin JA, Fridlyand J, Kageshita T, et al. Distinct sets of genetic alteration in melanoma. N Engl J Med 2005; 353: 2135-47.

11. Bauer J, Büttner P, Murali R, Okamoto I, Kolaitis NA, Landi MT, Scolyer RA, Bastian BC. BRAF mutations in cutaneus melanoma are independently associated with age, anatomic site of the primary tumor, ant the degree of solar elastosis at the primary tumor site. Pigment Cell Melanoma Res 2011; 24: 345-351.

12. Romano E, Schwartz GK, Chapman PB, Wolchock JD, Carvajal RD. Treatment implications of the emerging molecular classification system for melanoma. Lancet Oncol 2011; 12: 913-22.

13. Arkenau HT, Kefford R, Long GV. Targeting BRAF for patients with melanoma. Br J Cancer 2011; 104: 392-8.

14. Sosman JA, Kim KB, Schuchter L, et al. Survival in BRAF V600-mutant advanced melanoma treated with vemurafenib. N Engl J Med 2012; 366: 707-14

15. Chapman PB, Hauschild A, Robert C, et al.; BRIM-3 Study Group. Improved survival with vemurafenib in melanoma with BRAF V600E mutation. N Engl J Med 2011; 364: 2507-16.

16. Chapman PB, Hauschild A, Robert C et al. Updated overall survival (OS) results for BRIM-3, a phase III randomized, open-label, multicenter trial comparing BRAF inhibitor vemurafenib (vem) with dacarbazine (DTIC) in previously untreated patients with BRAFV600E-mutated melanoma. J Clin Oncol 2012; 30 (suppl): abstr. 8502.
17. Larkin JMG, Oueirolo P, Aranc AM et al. An open-label, multicenter safety study of vemurafenib (PLX4032, RO5185426) in patients with metastatic melanoma. J Clin Oncol 2012; 30 (suppl): abstr. 8517.

18. Hauschild A, Grob JJ, Demidov JV, et al. Phase III, randomized, openlabel, multicenter trial (BREAK-3) comparing the BRAF kinase inhibitor dabrafenib (GSK2118436) with dacarbazine (DTIC) in patients with BRAFV600E-mutated melanoma. J Clin Oncol 2012; 30 (suppl): abstr. LBA8500^.

19. Kirkwood JM, Long GV, Trefzer U, et al. BREAK-MB: A phase II study assessing overall intracranial response rate (OIRR) to dabrafenib (GSK2118436) in patients (pts) with BRAF V600E/k mutation-positive melanoma with brain metastases (mets). J Clin Oncol 2012; 30 (suppl): abstr. 8501.

20. Kudchadkar R, Paraiso KH, Smalley K. Targeting mutant BRAF in Melanoma. Current status and future development of combination therapy strategies. Cancer J 2012; 18: 124-31.

21. Flaherty KT, Robert C, Hersey P, et al. METRIC phase III study: Efficacy of trametinib (T), a potent and selective MEK inhibitor (MEKi), in progression-free survival (PFS) and overall survival (OS), compared with chemotherapy $(\mathrm{C})$ in patients (pts) with $\mathrm{BRAF}_{\mathrm{V} 600 \mathrm{E} / \mathrm{K}}$ mutant advanced or metastatic melanoma (MM). J Clin Oncol 2012; 30 (suppl): abstr. LBA8509.

22. Paraiso KH, Fedorenko IV, Cantini LP, et al. Recovery of phosphorERK activity allows melanoma cells to escape from BRAF inhibitor therapy. Br J Cancer 2010; 102: 1724-30.

23. Johannessen CM, Boehm JS, Kim SY, et al. COT drives resistance to RAF inhibition through MAP kinase pathway reactivation. Nature 2010; 468: 968-72.

24. Wagle N, Emery C, Berger MF, et al. Dissecting therapeutic resistance to inhibition in melanoma by genomic profiling. J Clin Oncol 2011; 29: 3085-96.

25. Poulikakos PI, Persuad Y, Janakiraman M, et al. RAF inhibitor resistance is mediated by dimerazation of aberrantly spliced BRAF (V600E). Nature 2011; 480: 387-90.

26. Weber JS, Flaherty KT, Jeffrey R. InfanteUpdated safety and efficacy results from a phase I/II study of the oral BRAF inhibitor dabrafenib (GSK2118436) combined with the oral MEK 1/2 inhibitor trametinib (GSK1120212) in patients with BRAF-naive metastatic melanoma. J Clin Oncol 2012; 30 (suppl): abstr. 8510.

27. Kalinsky K, Lee SJ, Lawrence DP, et al. A phase II trial of dasatinib in patients with unresectable locally advanced or stage IV mucosal, acral, and solar melanomas: An Eastern Cooperative Oncology Group study (E2607). J Clin Oncol 2012; 30 (suppl): abstr. 8522.

28. http://www.clinicaltrials.gov/ct2/results?term=NCT01028222.

29. Hodi FS, O'Day SJ, McDermott DF et al. Improved survival with ipilimumab in patients with metastatic melanoma. N Engl J Med 2010; 363: 711-23.

30. Robert C, Thomas L, Bondarenko I, et al. I pilimumab plus dacarbazine for previously untreated metastatic melanoma. N Engl J Med 2011; 364: 2517-26.

31. Maio M, Lebbé C, Sileni VCh, et al. Long-term survival in advanced melanoma patients treated with ipilimumab at $10 \mathrm{mg} / \mathrm{kg}$ : ongoing analyses from completed phase II Trials. ESMO Congress 2009; poster 116.

32. Maio M, Lebbé C, Neyns B, et al. Three-year survival rates for patients with advanced melanoma who received ipilimumab at $10 \mathrm{mg} / \mathrm{kg}$ in Phase II Trials. Congress - Perspectives in Melanoma XIV, Amsterdam 2010; poster P-0020.

33. Wolchok JD, Neyns B, Linette G, et al. Ipilimumab monotherapy in patients with pretreated advanced melanoma: a randomised, double-blind, multicentre, phase 2, dose-ranging study. Lancet Oncol 2010; 11: 155-64.

34. O’Day SJ, Maio M, Chiarion-Sileni V, et al. Efficacy and safety of ipilimumab monotherapy in patients with pretreated advanced melanoma: a multicenter single-arm phase II study. Ann Oncol 2010; 21: 1712-17.

35. O’Day SJ, Maio M, Chiarion-Sileni V, et al. A randomized,double-blind, placebo-controlled, phase II study comparing the tolerability and efficacy of ipilimumab administered with or without prophylactic budesonide in patients with unresectable stage III or IV melanoma. Clin Cancer Res 2009; 15: 5591-8.

36. Kähler KC, Hauschild A. Treatment and side effect management of CTLA-4 antibody therapy in metastatic melanoma. J Dtsch Dermatol Ges 2011; 9: 277-86. 
37. Lebbé C. Perspectives in Melanoma XII 2008; abstr. O-015.

38. Hersey P, Smalley KS, Weeraratna A, et al. Meeting report from the 7th International Melanoma Congress, Sydney, November, 2010. Pigment Cell Melanoma Res 2011; 24: 1: e1-15.

39. Weber J. Overcoming immunologic tolerance to melanoma: targeting CTLA-4 with ipilimumab (MDX-010). Oncologist 2008; 13 Suppl 4: $16-25$

40. Downey SG, Klapper JA, Smith FO, et al. Prognostic factors related to clinical response in patients with metastatic melanoma treated by CTL-associated antigen-4 blockade. Clin Cancer Res 2007; 13 (22 Pt 1): 6681-8.

41. Weber J. Ipilimumab: controversies in its development, utility and autoimmune adverse events. Cancer Immunol Immunother 2009; 58: 823-30.

42. Hamid O, Urba WJ, Yellin M. et al. Kinetics of response to ipilimumab (MDX-010) in patients with stage III/IV melanoma. J Clin Oncol 2007; 25 (Suppl. 25): abstr. 8525.

43. Wolchok J. Cancer Immunology \& Immunotherapy. XVIth Meeting of the Cancer Research Institute 2008; abstr. S25.

44. O Hamid, WJ Hwu, JM Richards et al. Ipilimumab (Ipi) expanded access program (EAP) for patients (pts) with stage III/IV melanoma: 10 mg/kg cohort interim results. J Clin Oncol 2012; 30 (suppl): abstr. 8508 .

45. http://www.clinicaltrials.gov/ct2/show/NCT01515189?term=ipilimumab+melanoma+10+mg+3+mg\&rank=1.

46. Harding JJ, Lacouture ME, Pulitzer M, et al. Hypersensitivity skin reac tions in melanoma patients treated with vemurafenib after ipilimumab therapy. J Clin Oncol 2012; 30 (suppl): abstr. 8515.

47. Ribas A, Hodi FS, Kurland JF, et al. CA184-161: A phase I/II trial of vemu rafenib and ipilimumab in patients with BRAF V600 mutation-positive metastatic melanoma. J Clin Oncol 2012; 30 (suppl): abstr. TPS8603.

48. Margolin K, Ernstoff MS, Hamid O, et al. Ipilimumab in patients with melanoma and brain metastases: an open-label, phase 2 trial. Lancet Oncol 2012; 13: 459-65.

49. Maio M, Testori A, Ascierto PA, et al. The NIBIT-M1 trial: Activity of ipilimumab plus fotemustine in patients with melanoma and brain metastases. J Clin Oncol 2012; 30 (suppl): abstr. 8529.

50. Kirkwod JM, Lorigan P, Hersey P, et al. A phase II trial of tremelimumab (CP-675,206) in patients with advanced refractory or relapsed melanoma. J Clin Oncol 2008; (suppl. 26): abstr. 9023.

51. Ribas A, Hauschild A, Kefford R, et al. Phase III, open-label, ran domized, comparative study of tremelimumab (CP-675,206) and chemotherapy (temozolomide [TMZ] or dacarbazine [DTIC]) in patients with advanced melanoma. J Clin Oncol 2008; (suppl. 26): abstr. LBA9011.

52. Tarhini AA, Moschos SS, Schlesselman JJ, et al. Phase II trial combination biotherapy of high-dose interferon alfa- $2 b$ and tremelimumab for recurrent inoperable stage III or IV melanoma. J Clin Oncol 2008; (suppl. 26): abstr. 9009

53. Butte MJ, Keir ME, Phamduy TB, Sharpe AH, Freeman GJ. Programmed death-1 ligand interacts specifically with the B7-1 costimulatory molecules to inhibit T cell responses. Immunity 2007; 27: 111-22.

54. Hodi FS, Sznol M, McDermott DF, et al. Clinical activity and safety of anti-PD-1 (BMS-936558, MDX-1106) in patients with advanced melanoma (MEL). J Clin Oncol 2012; 30 (suppl): abstr. 8507.

55. Kudchadkar RR, Gallenstein D, Martinez AJ, et al. Phase I trial of extended-dose anti-PD-1 antibody BMS-936558 with a multipeptide vaccine for previously treated stage IV melanoma. J Clin Oncol 2012; 30 (suppl): abstr. 8582.

56. Sznol M, Hodi FS, Margolin K. Phase I study of BMS-663513, a fully human anti-CD137 agonist monoclonal antibody, in patients (pts) with advanced cancer (CA). J Clin Oncol 2008; (suppl): abstr. 3007.

57. http://www.clinicaltrials.gov/ct2/show/NCT00612664?term=BMS $663513 \&$ rank $=6$

58. Vonderheide RH, Flaherty K, Khalil M, et al. Clinical activity and immune modulation in cancer patients treated with CP-870,893, a novel CD40 agonist monoclonal antibody. J Clin Oncol 2006; 25 876-83.

59. http://www.clinicaltrials.gov/ct2/show/NCT00607048?term=CP$870 \% 2$ C893\&rank=3.
60. Morton DL, Mozzillo N, Thompson JF, et al. An international, randomized, phase III trial of bacillus Calmette-Guerin (BCG) plus allogeneic melanoma vaccine (MCV) or placebo after complete resection of melanoma metastatic or regional or distant sites. J Clin Oncol 2007; (suppl): abstr. 8508.

61. Vaishampayan U, Abrams J, Darrah D, Jones V, Mitchell MS. Active immunotherapy of metastatic melanoma with allogeneic melanoma lysates and interferon alpha. Clin Cancer Res 2002; 8: 3696-6701.

62. Mackiewicz J, Mackiewicz A. Gene-modified cellular vaccines: technologic aspects and clinical problems. Transplant Proc 2010; 42: 3287-92.

63. Mackiewicz A, Mackiewicz J, Wysocki PJ, et al. Long-term survival of high-risk melanoma patients immunized with a hyper-IL-6modified allogeneic whole-cell vaccine after complete resection. Expert Opin Investig Drugs 2012; 21: 773-83.

64. Eggermont AMM, Suciu S, Samtinam M, et al. Adjuvant therapy with pegylated interferon $\alpha$-2b versus observation in resected stage III melanoma. Final results of EORTC 18891, a randomized phase 3 trial. Lancet 2008; 372: 117-26.

65. Eigentler TK, Radny P, Hauschild A, et al. Adjuvant treatment with vindesine in comparison to observation alone in patients with metastasized melanoma after complete metastasectomy: a randomized multicenter trial of the German Dermatologic Cooperative Oncology Group. Melanoma Res 2008; 18: 363-8.

66. Bystryn JC, Zeleniuch-Jacquotte A, Oratz R, et al. Double-blind trial of polyvalent, shed-antigen, melanoma vaccine. Clin Cancer Res 2011: 1882-7.

67. Pennacchioli E, Gandini S, Verrecchia F, et al. Surgery in stage IV melanoma patients: Results from a single institution. J Clin Oncol 2012; 30 (suppl): abstr. e19035.

68. Banchereau J, Steinman RM. Dendritic cells and the control of immunity. Nature 1998; 392: 245-52.

69. Hart DN. Dendritic cells: unique leukocyte populatons which control the primary immune response. Blood 1997; 90: 3245-87.

70. Schadendorf D, Ugurel S, Schuler-Thurner B, et al. Dacarbazine (DTIC) versus vaccination with autologous peptide-pulsed dendritic cells (DC) in first-line treatment of patients with metastatic melanoma: a randomized phase III trial of the DC study group of the DeCOG. Ann Oncol 2006; 17: 563-70.

71. Engel-Noerregaard L, Hansen TH, Andersen MH, Straten PT, Svane IM. Review of clinical studies on dendritic cell-based vaccination of patients with malignant melanoma: assessment of correlation between clinical response and vaccine parameters. Cancer Immunol Immunother 2009; 58: 1-14.

72. NN Petenko, IN Mikhaylova, GZ Chkadua, AY Baryshnikov, LV Demidov et al. Adjuvant dendritic cell (DC)-based vaccine therapy of melanoma patients. J Clin Oncol 2012; 30 (suppl): abstr. 2524

\section{Address for correspondence}

\section{Jacek Mackiewicz}

Department of Cancer Immunology

Chair of Medical Biotechnology

Poznan University of Medical Sciences

Greater Poland Cancer Centre

Garbary 15

61-866 Poznan

e-mail: jmackiewicz@biocontract.com

Submitted: $\quad 1.10 .2012$

Accepted: $\quad$ 20.10.2012 